\title{
Avaliação do processo de atendimento a pacientes portadores de doença crônico-degenerativa em uma unidade básica de saúde
}

\author{
Evaluation of management of patients with chronic degenerative diseases in a \\ primary health clinic
}

Arnaldo Sala *, Diane D. Cohen *, Ana S. W. Dalmaso *, Ȧngela M. M. Lima*, Ricardo R. Teixeira*

\begin{abstract}
SALA, A. et al. Avaliação do processo de atendimento a pacientes portadores de doença crônico-degenerativa em uma unidade básica de saúde. Rev. Saúde Pública, 27: 463-71, 1993. Avalia-se o desempenho de açōes de saúde desenvolvidas em uma unidade básica de saúde, relativas ao controle da hipertensão arterial sistêmica (HAS) enquanto estratégia de redução de morbi-mortalidade por doença cardiovascular baseada no "enfoque de risco". Estas açõcs estruturam-se a partir da deteç̧ão da hipertensão arterial na população adulta atendida no serviço e do controle dos níveis pressóricos nos indivíduos portadores de HAS, incluindo outros fatores de risco conhecidos, bem como tratamento de eventuais complicações. Analisaram-se 3.793 usuários que compareceram pelo menos uma vez à consulta médica no serviço de Assistência ao Adulto de um Centro de Saúde-Escola, do Município de São Paulo (Brasil), no período de $1^{\circ}$ de junho de 1990 a 31 de maio de 1991. Para cada um dos usuários foram considerados os diagnósticos realizados, bem como a concentração de cada modalidade de consulta realizada (pronto-atendimento e consulta agendada). Destes, 839 cram portadores de hipertensão arterial e/ou diabete e foram agrupados em quatro categorias: os exclusivamente hipertensos, os hipertensos com outra doença crônica associada (exceto diabete), os diabéticos e os diabéticos com hipertensão arterial. Os resultados deste estudo mostraram: 1) baixa cobertura de indivíduos hjpertensos e diabéticos $\mathrm{em}$ atendimento no serviço, quando se considera a população atendida pclo Centro de Saúde; 2) a existência de pacientes diagnosticados como hipertensos em consultas de pronto-atendimento, que não retornaram ao Centro de Saúde para seguimento médico programático, apontando para dificuldades na captação efetiva destes indivíduos. Esta "perda" deveu-se tanto a faltas dos pacientes às consultas agendadas para seu seguimento quanto ao não agendamento de consultas de seguimento por parte do serviço; 3) para os pacientes que aderiram ao seguimento, a concentração de consultas médicas e a concentração de faltas apresentaram números compatíveis com a proposta de agendamento trimestral; 4) a categoria dos exclusivamente hipertensos apresentou, quando comparada com as demais, menor concentração de consultas e maior proporção de faltas por consulta agendada. Discutem-se os limites das ações bascadas no "enfoque de risco" para controle de doenças crônico-degenerativas em população.
\end{abstract}

Descrilores: Avaliação de processos e resultados (cuidados de saúde). Hipertensão, prevenção. Avaliação de programas.

\section{Introdução}

Já há algum tcmpo, a magnitude das doenças cardiovasculares como causa de óbito c morbidade tem suscitado cstudos que relacionam certos fatores (tabagismo, alcoolismo, hipercolestcrolcmia, obesidade, "stress", diabetes melitus, hiper-

\footnotetext{
* Centro de Saúde-Escola Prof. Samucl B. Pessoa Departamento de Medicina Preventiva da Faculdade de Medicina da Univcrsidade de São Paulo - São Paulo, SP - Brasil
}

Separatas/Reprints: A. Sala - Av. Vital Brasil, 1490 - 05503. 000 - São Paulo, SP - Brasil

Edição subvencionada pela FAPESP. Processo Medicina 93/ 0208-5. tensão artcrial c outros), ao aparecimento dessas doenças ${ }^{5}$.

No intuito de tentar evitar a morte precoce e a morbidade por essas citadas doenças, em sua maior parte incapacitantes, têm-se formulado duas estratégias básicas: a do "cnfoque de risco" c a do "enfoque populacional"7. O primeiro propõe o controle de exposição a fatores de risco $\mathrm{cm}$ indivíduos com alto risco para doenças carcliovascularcs (DCV), cm trabalho rcalizado individuo a indivíduo. Já o "cnfoque populacional" pretende alcançar a redução dos fatores de risco na população, rcalizando-se a abordagem ao nível do colctivo. Ambas as estratégias têm como pressuposto que a supressão de um ou mais fatores de risco acarretaria diminuição da mortalidade e morbidade por DCV. 
Em dezembro de 1986, o Ministério da Saúde, pela sua Divisão Nacional de Doenças CrônicoDegenerativas, lançou as "Bases Estratégicas e Operacionais" para o controle das DCV, em documento ${ }^{6}$ onde faz uma breve avaliação do trabalho realizado naquele ano e expóe sua proposta de atuação. $O$ documento prevê que a abordagem do problema seja feita através dos dois enfoques, o de risco e o populacional.

O trabalho de prevenção pela "estratégia populacional" scria realizado por meio de campanhas anti-fumo, a favor dos exercícios físicos e da dicta mais equilibrada. Já o trabalho de prevenção pela "estratégia de alto risco" seria realizado pela assistência médica prestada aos individuos de alto risco, especialmente hipertensos, com a deteç̧ão e controle dos principais fatores de risco, tais como fumo, obesidade, hipercolesterolemia, entre outros.

A avaliação de programa de controle de DCV, bascado no "enfoque de risco", deveria conter, cm última instância, apreciação de scus resultados finais sobre o comportamento desse grupo de doenças na população, finalidade última do citado programa. Contudo, algumas dificuldades operacionais e conccituais dificultam, na prática, a realização de tal procedimento. Primciramente, scria necessário obter cobcrtura suficientemente expressiva de individuos com risco para $\mathrm{DCV}$ para que sc obscrvassc uma modificação significaliva no perfil cpidemiológico; isto impediria uma avaliação ainda na fase de implantação do programa. Em segundo lugar, o grupo de indivíduos com risco para DCV não produz a maioria dos casos de doença, na medida em que é um grupo numericamente bem menor que o dos indivíduos de baixo risco (e portanto não objeto do programa); desta forma, a melhoria dos indicadores de morbi-mortalidade por DCV scria scmprc parcial. Em tercciro lugar, a oblenção desse cfeito descjado na população não se faria de imcdiato, mas após um período de vários anos, o que dificultaria o processo de rcorientação contínua das açõcs. Essc tipo de avaliação de resultados finais não permitiria visualizar o desempenho dos elementos constitutivos do processo de atendimento desses individuos de risco, não possibilitando, assim, localizar no conjunto das ações desenvolvidas, aquelas que necessitam prioritariamente de alteraçōes.

Assim, uma metodologia de avaliação que possa apreender os clementos do trabalho que envol$v \mathrm{em}$ o atendimento desses indivíduos de risco para DCV parece ser mais pertinente, principalmente quando se considera uma fase ainda de implantação do programa (de ordenamento do trabalho e de extensão de cobcrtura). Esta pers- pectiva avaliatória parte do suposto de que se 0 processo de trabalho não for adequado, do ponto de vista de sua coerência com os objetivos finais a serem alcançados, os resultados finais (controle da DCV na população) não devem estar sendo alcançados. Embora não podendo apreender esses resultados finais, essa avaliação permite identificar pontos no conjunto do trabalho que necessitcm modificações.

A avaliaçấo do processo de trabalho não deve ser confundida com avaliação de processo, tal como proposta por Donabedian ${ }^{3}$. Embora seja possivel identificar algumas semelhanças entre os respectivos meios de avaliação, tratam-se de perspectivas conceituais distintas que produzem, conseqüentemente, uma visibilidade diversa das ações de cuidado à saúde. A perspectiva proposta por Donabedian ${ }^{3}$ busca avaliar, em última instância, a racionalidade das açőes, tomando como elemento invariante, a cada momento, o conhecimento médico estabelecido. Nesses termos, a avaliação deve scr capaz de identificar possíveis (e prováveis) desvios $\mathrm{e}$ distorções na aplicação do conhecimento médico disponivel, no momento da efetivação do cuidado à saúde.

$\mathrm{Na}$ perspectiva proposta no presente texto, a da avaliação do processo de trabalho ${ }^{8}$, busca compreender as relaçōes que se estabelecem, no processo de trabalho em saúde, entre os elementos que o constitucm: objeto do trabalho, meios de trabalho (onde se inclucm, alćm dos instrumentos materiais, o conhecimento clínico e o conhecimento epidemiológico), e agentes do trabalho; estes são determinados, em última instância, pela necessidade social de obtenção dos produtos desse processo de trabalho. Nesses tcrmos, a avaliação deve reconhecer quais são essas necessidades (ou, pelo menos, qual é sua expressão hegemônica), e verificar se o processo efelivamente realizado é adequado à satisfação dessas necessidades sociais. É evidente que a satisfação cfetiva dessa necessidade só pode scr aprecndida pelo próprio conjunto social que, ao fazê-lo, reitcra, modifica ou substitui aquele processo por outro*.

Deste modo, três enfoques aparecem como pertinentes para a avaliação do processo de trabalho relativo a ações programáticas dirigidas ao controle de DCV: avaliação da cobertura, avaliação do uso do serviço, e avaliação do conteúdo das ações, dos quais apenas os dois primeiros scrão considerados enquanto objeto da presente investigação.

\footnotetext{
* Para aprofundamento da questão rclativa ao processo de
} trabalho, ver Gonçalves 4 . 
$\mathrm{Na}$ avaliação da cobertura considera-se, fundamentalmente, a relação entre a demanda que cletivamente compareceu ao serviço para atendimento no programa e a demanda potencial constituída pclos indivíduos suscetiveis ao desenvolvimento de DCV, pertencentes à área de abrangência do serviço.

$\mathrm{Na}$ avaliação do uso do serviço, a questão central é a da verificação da ocorrência de uma concentração mínima necessária de determinadas atividades (adequada para cada situação de ação programática), incidindo sobre cada indivíduo, tendo como pressuposto que se o uso do serviço não for adequado (baixa concentração das atividades por paciente), então o conjunto do trabalho deve scr insuficiente para a detecção de fatores de risco, bem como para o controle da doença já instalada.

O tcrceiro ponto, não considcrado no desenho da presente investigação, consiste na avaliação do conteúdo das ações, que envolve a análise de múltiplos aspectos, no sentido da verificação da clicácia dos processos de diagnóstico, de identificação de fatores de risco em cada indivíduo e dos processos terapĉuticos. Esta eficácia não deve ser entendida apenas no sentido restrito aos parâmetros técnicos definidos pelo conhecimento médico; scu sentido, pois, deve scr ampliado para comprecnder o conjunto do trabalho, $\mathrm{cm}$ perspectivas complementares que envolvem desde as demandas e expectativas do usuário $\mathrm{cm}$ rclação ao atendimento, a aderência dos agentes (médicos, cnfermagem e outros) envolvidos no atendimento, os processos diagnósticos e tcrapêuticos, até a articu. lação entre as diferentes atividades que compãcm o conjunto das ações programáticas.

O presente estudo tem por objelivo avaliar o desempenho de um programa de controle de doenças crônico-degencrativas opcrado concretamente em uma unidade básica de saúde que $1 \mathrm{em}$ suas ações oricntadas a partir da integração entre práticas programáticas e assistência médica à demanda espontânea ${ }^{9}$. Esta avaliação, realizada a partir de informações de prontuário dos individuos atendidos durante o periodo do estudo, vai enfocar questões relativas à cobertura na população adscrita à unidade, à captação da demanda de "indivíduos de risco" para DCV c ao uso do scrviço.

\section{Material e Método}

O matcrial cmpírico ć refcrente aos usuários de um Centro de Saúde-Escola (CSE) do Município de São Paulo, atendidos no sctor de assistência ao adulto, no período compreendido entre primeiro de junho de 1990 e 31 de maio de 1991, enfocando tanto as modalidades de atendimento a que foram submetidos os pacientes, quanto aos diagnósticos relativos às doenças crônicas, objetos de atividade programática. Esse Centro está localizado no bairro do Butantã, e atende a uma população geograficamente delimitada, constituída por 47.016 habitantes, em 1991 (Censo 1991*, IBGE, dados por setor censitário), da qual estima-se que $60,4 \%$ têm 20 anos de idade ou mais.

\section{Centro de Saúde-Escola}

No CSE estudado, as açõcs de controle de DCV seguem a padronização do Ministério da $S^{2} u_{d e}^{5}$, no que diz respeito ao cuidado através da "estratégia do alto risco".

A organização das atividades no setor de assistência ao adulto do CSE foi conccbida como subordinada à questão do envelhecimento ${ }^{1}$. No cntanto, o cuidado aos pacientcs portadores de doenças crônico-degencrativas foi implantado $\mathrm{cm}$ curto espaço de tcmpo, ficando as açõcs dirigidas ao conjunto da população que envelhece com um ritmo mais lento de implantação, dada a natureza de scu próprio objeto. Deste modo, na fase inicial, a organização das atividades aparentava possuir dois programas distintos - 0 de crônicos e o de idosos - que apenas em período posterior ao presente estudo apresentam-se, de fato, integrados.

A organização dos cuidados dirigidos aos pacientes portadores de docnça crônico-degencrativa possui, no CSE, dois momentos principais: o atendimento não programático e o acompanhamento médico programático. O primciro, ou pronto-atendimento (PA), tem dois objetivos básicos: o atendimento médico imediato aos pacientes que procuram o serviço sem consulta agendada c com queixa e a captação ativa de demanda para as atividades programáticas realizadas no serviço (Programa de Assistência à Saúde no Envelhecimento, incluindo o Programa de Controle de Doenças Crônicas, Programa de Assistência à Saúde do Adolescente e Programa de Assistência à Saúde da Mulher).

O acompanhamento médico programático $\mathrm{cn}$ volve, basicamente, consultas médicas agendadas e atendimentos $\mathrm{cm}$ grupo realizados com a participação de pessoal auxiliar de enfermagem.

Especificamente para o Programa de Controle de Doenças Crônicas, a captação ativa de demanda É realizada da seguinte forma: mede-se a pressão artcrial de todos os indivíduos que passam pelo pronto-atendimento, independentemente da queixa

\footnotetext{
* Dados inćditos.
} 
que apresentem. Tambćm é verificada a pressão arterial de todos os pacientes que fazem seguimento programático no serviço, em qualquer um dos programas, pclo menos uma vez por ano.

O paciente considerado hipertenso é encaminhado para a consulta de início do seguimento, uma consulta médica que pretende fazer levantamento de outros fatores de risco aos quais o indivíduo estaria submetido. A seguir, o paciente cntra numa rotina de seguimento, o que significa dizer que ele terá uma consulta médica agendada, como norma gcral, trimestralmente, além de realizar exames complementares, anualmente, com o objetivo principal de detectar complicações bem como diagnosticar, de forma precoce, alguns outros fatores de risco para DCV.

\section{Coleta de Dados}

No CSE estudado, desde 1989, colctam-sc dados dos prontuários de todos os atendimentos realizados, como também dos faltosos ao agendamento, que são organizados $\mathrm{cm}$ arquivo informatizado, através da utilização do software FoxBASE+. Os dados contidos nesse arquivo são: scxo; idade; tipo ou modalidade de atendimento (PA, início de seguimento, retorno agendado, atendimento $\mathrm{cm}$ grupo $\mathrm{e}$ atendimento de enfermagem); nome do programa (Mulher, Gestante, Crônicos, Adolcscentes e Outros); comparecimento/falta ao atendimento; diagnóstico de doença crônica (HA (hipertensão arterial), DM (diabctes mclito), cardiopatia, DPOC (doença pulmonar obstrutiva crônica), obesidade e epilepsia) presentes nas consultas realizadas no período.

Para se proceder à análise dos usuários do setor, no período de um ano, utilizaram-sc dados dos atendimentos realizados (todos os tipos) e das faltas ocorridas no periodo.

Assim, a partir de um processamento do arquivo de atendimentos, foi claborado um outro arquivo, de usuários do setor de assistência ao adulto. Este processamento consistiu, basicamente, na contagem dos atendimentos $\mathrm{cm}$ cada uma das modalidades a que se submetcu cada usuário, assim como na vcrificação da presença de cada um dos diagnósticos de docnça crônica $\mathrm{cm}$ pclo mcnos uma das consultas realizadas no período. Esse arquivo de usuários contém os seguintes dados, que foram utilizados dirctamente para a produção dos resultados da presente avaliação:

1 - idadc;

2 - sexo;

3 - concentração de atendimentos realizados (todos os tipos) nos programas de Mulher, Gestante, Crônicos c Outros;
4 - concentração de atendimentos realizados (todos os programas) nos tipos de atendimento, consulta de PA, consulta agendada, atendimento de enfermagem e atendimento em grupo;

5 - concentração de consultas médicas do programa de Crônicos;

6 - concentração de faltas em consultas médicas agendadas de pacientes do programa de Crônicos;

7 - diagnóstico, onde os pacientes foram agrupados em 5 categorias nosológicas, a partir da presença ou ausência de cada um dos 6 diagnósticos inicialmente considerados, $\mathrm{em}$ pclo menos uma das consultas realizadas, com a seguinte nomenclatura:

- HA EXCLUSIVO: portador exclusivo de hipertensão

- HA: portador de hipertensão com outra docnça crônica, exceto diabcte

- DM: portador de diabcte com outra doença crônica, exceto hipertensão

- HA+DM: hipertenso e diabético, com ou sem outras doenças crônicas

- NÃO CRÔNICO: não portador de hipertensão, nem de diabete, com ou sem outros diagnósticos de doença crônica

8 - uso do serviço, categorizado em usuário cxclusivo do PA e em usuário de consulta agendada (quando foi realizada pelo menos uma consulta em qualquer um dos programas, tendo ou não sido realizadas consultas de PA).

Deste modo, de um total de 16.809 registros de atendimentos ou de faltas, foi organizado um arquivo com 3.793 usuários matriculados que compareceram pelo menos uma vez a qualquer modalidade de consulta.

O processamento desse arquivo de usuários, para a claboração das tabelas de distribuição de frcqüência c para a análise estatística, foi realizado através do software EPINFO. Foi utilizada análise de variância unifatorial para os dados numéricos com distribuição normal, e o teste de Kruskal-Wallis nas situações em que estes não possucm distribuição normal. Para análise dos dados categóricos foi utilizado o teste de Chi quadrado.

A análise da cobcrtura tem como referência a estimativa de prevalência de hipertensão $\operatorname{arterial}^{5} \mathrm{e}$ diabete ${ }^{2}$ na população gcral.

A análise da utilização do serviço, considerada a partir de cada uma das quatro categorias de pacientes agrupados pela sua situação diagnóstica, percorrcu inicialmente a concentração de atendimentos em consultas agendadas. A expectativa do 
Programa é de quatro consultas programáticas, agendadas por ano, para os indivíduos que iniciaram o seguimento antes do período de estudo, e um númcro baixo de consultas de pronto-atendimento, na medida em que o atendimento programático deve resolver a maior parte dos problemas de saúde daqucle individuo, sejam estes problemas rclacionados ou não às doenças crônico-degenerativas, objeto do programa. A observação das faltas ocorridas no período completa a análise inicial da utilização do serviço naquilo que se pode denominar como aderência ou assiduidade ao atendimento. No conjunto de todos os atendimentos realizados no setor de atendimento ao adulto do CSE, $\mathrm{tcm}$-se uma proporção de aproximadamente $30 \%$ de faltas sobre o total de agendamentos*.

A análise da utilização de serviço completa-se com a verificação da presença de atendimentos programáticos, durante o periodo de estudo, contra a presença exclusiva de consultas de prontoatendimento, em cada um dos quatro grupos de pacientes considerados. Procura-se verificar não só a existência de um padrão homogênco na distribuição de cada grupo de pacientes, scgundo a presença de atendimento programático, mas também o volume de indivíduos que permanece exclusivamente no pronto-atendimento, o que revelaria dificuldades na captação destes para a assistência programática.

\section{Resultados}

Os dados apresentados permitem considerar as seguintes questōes:

\section{Cobertura}

A cobertura de hipertensos c diabéticos é baixa, cstando ao redor de $2,7 \%$ c $0,7 \%$, respectivamentc, quando sc considcra a população adulta (indivíduos com 20 anos de idade ou mais) residente na área do CSE. Mesmo considerando que um segmento dessa população lenha accsso a outros scrviços de assistência médica além do CSE, ć provåvel que um grupo significativo de hiperiensos e de diabéticos não estejam sendo submetidos a nenhum controle de saúde, ou mesmo não tcnham sido nem objeto de diagnóstico.

A Tabcla 1 mostra a prevalência de indivíduos portadores de "doenças crônicas" na população adulta usuária do serviço, mostrando prevalência acima de $50 \%$ na faixa etária com 50 anos e mais.

* Dados do Bolctim de Produção do CSE - documento in. terno.
Tabela 1. Taxa de prevalência anual das "Doenças Crônicas" por 100 pacientes, em 3.793 pacientes atendidos no setor de assistência ao adulto do Centro de Sáude-Escola, segundo grupo etário. Periodo de junho de 1990 a maio de 1991.

\begin{tabular}{lccc}
\hline Diagnóstico* & $N$ & \multicolumn{2}{c}{ Faixa Etária } \\
\cline { 2 - 4 } & & $12-49$ & 50 e mais \\
\hline DM & 62 & 0,5 & 5,3 \\
HA & 177 & 1,9 & 13,9 \\
HA-DM** & 137 & 0,7 & 13,3 \\
HA excl. & 461 & 6,8 & 30,1 \\
\hline Total \% & 22,0 & 9,9 & 62,6 \\
Número & 837 & 289 & 548 \\
\hline
\end{tabular}

- $D M=$ diabete com ou sem outra doença crónica (exclui HA).

$H A=$ hipertensão com outra doença crônica (exclui diabetes)

HA-DM = hipertensāo e diabetes com ou sem outra doença crônica.

HA excl. = HA exclusiva.

* Houve perda de 2 casos sem informação sobre idade.

\section{Uso do serviço}

Considcrados apenas os pacientes inscritos no Programa antes do período estudado, os dados sobre concentração de atendimentos de Hipertensos, Diabéticos e IIipertensos Diabéticos mostra médias compativeis com a proposta de agenda-

Tabela 2. Distribuição percentual de pacientes crônicos, segundo concentraçāo de comparecimentos em consultas agendadas e situação diagnósticada, em pacientes inscritos no programa até o início do periodo estudado*, junho de 1990 a maio de 1991.

\begin{tabular}{lcrrrr}
\hline $\begin{array}{l}\text { Concentração } \\
\text { de } \\
\text { comparecimentos }\end{array}$ & \multicolumn{5}{c}{ Diagnóstico** } \\
\cline { 2 - 6 } & DM & HA & HA-DM & HA Exc & Total \\
\hline 1 e 2 & 20,0 & 15,2 & 8,8 & 38,3 & 25,8 \\
3 a 5 & 40,0 & 57,1 & 46,1 & 45,6 & 47,9 \\
6 e 7 & 22,9 & 18,8 & 32,4 & 13,3 & 19,1 \\
8 e + & 17,1 & 8,9 & 12,7 & 2,8 & 7,2 \\
\hline Total \% & 100,0 & 100,0 & 100,0 & 100,0 & 100,0 \\
Número & 35 & 112 & 102 & 248 & 497 \\
Média & 5,2 & 4,5 & 5,2 & 3,4 & 4,2 \\
D.P. & 3,3 & 2,0 & 2,1 & 2,0 & 2,3 \\
\hline
\end{tabular}

$p<0,05$

Exclui 234 pacientes que fizeram inscrição durante o periodo do estudo e 108 pacientes não inscritos

- $D M=$ diabete com ou sem outra doença crónica (exclui HA).

$H A=$ hipertensão com outra doença crônica (exclui diabetes).

HA-DM = hipertensão e diabeles com ou sem outra doença crônica.

HA excl. = HA exclusiva.

Nota: Existe diferença estatisticamente significativa entre o grupo de HA excl. e os demais crónicos. 
mento trimestral de consulta, com pequena proporção de pacientes com apcnas uma consulta programática (Tabcla 2). Os Hipertensos Exclusivos apresentam menor número de consultas realizadas no período, sugerindo aderência ou assiduidade significativamente menor à proposta de agendamento trimestral*.

Desta forma, pode-se concluir que o uso do scrviço, indicado pela concentração de consultas do Frograma (Tabcla 2) e de PA (Tabela 3), pode scr considcrado como adequado, exceto para o grupo dos hipertensos exclusivos.

Tabela 3. Distribuição percentual de pacientes crônicos, segundo concentração de comparecimentos ao prontoatendimento e situação diagnóstica. Período de junho de 1990 a majo de 1991.

\begin{tabular}{lccccc}
\hline $\begin{array}{l}\text { Concentração } \\
\text { de } \\
\text { comparecimentos }\end{array}$ & \multicolumn{5}{c}{ Diagnóstico* $^{*}$} \\
\cline { 2 - 6 } & DM & HA & HA-DM & HA Exc & Total \\
\hline 0 & 29,0 & 40,1 & 38,1 & 34,3 & 35,8 \\
1 & 27,4 & 27,1 & 32,4 & 38,6 & 34,3 \\
2 & 25,8 & 17,0 & 15,8 & 15,6 & 16,7 \\
3 e + & 17,8 & 15,8 & 13,7 & 11,5 & 13,2 \\
\hline Tolal \% & 100,0 & 100,0 & 100,0 & 100,0 & 100,0 \\
Número & 62 & 177 & 139 & 46.1 & 839 \\
Média & 1,5 & 1,3 & 1,2 & 1,1 & 1,2 \\
D.P. & 1,5 & 1,5 & 1,5 & 1,2 & 1,3 \\
\hline
\end{tabular}

$p<0,05$

$D M=$ diabete com ou sem outra doença crônica (exclui HA).

HA = hipertensão com outra doença crōnica (exclui diabetes).

HA-DM = hipertensão e diabetes com ou sem outra doença crônica.

HA excl. = HA exclusiva.

Nota: As diferenças não são estatisticamente significativas.

Fode-sc afirmar, no entanto, que a menor "aderência" dos pacientes Ilipertensos Exclusivos não ć compensada por consultas mais freqüentes ao $P A$. Trata-sc, pois, de um grupo de indivíduos que cfelivamente utiliza menos o scrviço (Tabcla 3 ). Da mesma forma, esses pacientes possuem maior índice de falta às consultas nédicas do Programa (Tabcla 4).

O grupo de pacientes Ilipertensos Exclusivos é constiluído por uma população significativamente mais jovem (Tabcla 5) do que a dos outros grupos de crônicos. Nesse grupo é que se observa quanti-

\footnotetext{
* Foram excluídos os pacientes inscritos no atendimento programático durante o período do presente estudo, na medida cm que, cm função de situaçōes clínicas bas. tante variadas, a proposta de agendamento trimestral não se aplica, cm geral, às primeiras consultas.
}

Tabela 4. Distribuição percentual de pacientes crônicos", segundo faltas por 100 agendamentos de consultas médicas e situação diagnosticada. Periodo de junho de 1990 a maio de 1991.

\begin{tabular}{lrrrrr}
\hline $\begin{array}{l}\text { Faltas } \\
\text { por } \\
\text { 100 agend. }\end{array}$ & \multicolumn{5}{c}{ Diagnóstico** } \\
\cline { 2 - 6 } & DM & HA & HA-DM HA Exc & Total \\
\hline O & 61,1 & 54,4 & 57,3 & 54,9 & 55,7 \\
1 a 14 & 5,5 & 7,6 & 9,6 & 1,6 & 4,8 \\
15 a 29 & 7,4 & 18,1 & 16,9 & 13,2 & 14,6 \\
30 a 49 & 16,7 & 12,9 & 12,5 & 11,9 & 12,5 \\
50 a 100 & 9,3 & 7,0 & 3,7 & 18,4 & 12,3 \\
\hline Total \% & 100,0 & 100,0 & 100,0 & 100,0 & 100,0 \\
Número & 54 & 171 & 136 & 370 & 731 \\
Média & 13,8 & 13,1 & 12,1 & 17,9 & 15,4 \\
D.P. & 21,5 & 17,0 & 17,0 & 23,3 & 20,9 \\
\hline
\end{tabular}

$p<0,05$

Exclui 108 pacientes não inscritos no programa (não possuem consultas agendadas

* $D M=$ diabete com ou sem outra doença crônica (exclui HA).

$\mathrm{HA}=$ hipertensāo com outra doença crônica (exclui diabetes).

HA-DM = hipertensão e diabetes com ou sem outra doença crônica.

$\mathrm{HA}$ excl. = HA exclusiva.

Nota: As diferenças encontradas são estatisticamente significativas.

Tabela 5. Distribuição percontual de pacientes crônicos, segundo idade e diagnóstico. Período de junho de 1990 a maio de 1991.

\begin{tabular}{lcrrrr}
\hline $\begin{array}{l}\text { Faixa } \\
\text { etária }\end{array}$ & \multicolumn{5}{c}{ Diagnóstico* } \\
\cline { 2 - 6 } & DM & HA & HA-DM** HA Exc & Total \\
\hline 12 a 19 & 3,2 & - & 0,7 & 0,7 & 0,7 \\
20 a 49 & 22,6 & 31,1 & 14,6 & 42,1 & 33,8 \\
50 a 59 & 29,0 & 23,7 & 26,3 & 23,6 & 24,5 \\
60 e + & 45,2 & 45,2 & 58,4 & 33,6 & 41,0 \\
\hline Total & 100,0 & 100,0 & 100,0 & 100,0 & 100,0 \\
Número & 62 & 177 & 137 & 461 & 837 \\
Média & 56,3 & 57,3 & 60,0 & 52,3 & 54,9 \\
D.P. & 16,2 & 12,9 & 11,2 & 13,7 & 13,7 \\
\hline
\end{tabular}

$p<0,05$

$D M=$ diabele com ou som outra doença crônica (exclui HA).

$H A=$ hipertensão com outra doença crôniça (exclui diabetes).

HA-DM = hipertensão e diabetes com ou sem outra doença crônica.

HA excl. = HA exelusiva.

* Há perda de informação de idade em 2 casos de HADM.

Nota: As diferenças observadas são estatisticamente significativas.

dade expressiva de indivíduos atendidos apenas $\mathrm{cm}$ PA (não tendo comparecido a nenhuma consulta do Programa) (Tabela 6), indicando que 0 grupo, portador apenas de um dos fatores de risco 
para DCV investigados pelo serviço, parece ter menor "adcrência" a uma proposta de controle de saúde, $\mathrm{cm}$ siluação de ausência relativa de sintomas, $\mathrm{cm}$ contraste com os pacientes nas outras categorias nosológicas. O tcrmo 'adcrência' rcfcre-se, na realidade, ao não comparecimento do indivíduo à consulta agendada, podendo implicar desde a negação total ou parcial da proposta de atendimento ofcrecida até questőes de dificuldades opcracionais para compareccr ao scrviço no dia $\mathrm{c}$ hora marcados. A Tabcla 7 mostra que a distribuição por

Tabela 6. Distribuiçăo percentual de pacientes crônicos segundo situação diagnóstica e uso do serviço. Periodo de junho de 1990 a maio de 1991.

\begin{tabular}{lcrrr}
\hline \multicolumn{5}{c}{ Uso do serviço } \\
\hline Diagnóstico* & $\begin{array}{c}\text { Consulta } \\
\text { agend.* }\end{array}$ & $\begin{array}{c}\text { Pronto-atend. } \\
\text { exclusivo** }\end{array}$ & $\begin{array}{c}\text { Total } \\
\%\end{array}$ & Número \\
\hline DM & 87,1 & 12,9 & 100,0 & 62 \\
HA & 96,6 & 3,4 & $-100,0$ & 177 \\
HA-DM & 97,8 & 2,2 & 100,0 & 139 \\
HA excl & 80,3 & 19,7 & 100,0 & 461 \\
\hline Tolal & 87,1 & 12,9 & 100,0 & 839 \\
\hline
\end{tabular}

- $D M=$ diabete com ou sem outra doença crônica (exclui HA).

$H A=$ hipertensão com outra doença crônica (exclui diabetes).

HA-DM = hipertensão e diabetes com o'd sem outra doença crônica.

HA excl. = HA exclusiva.

* Consulta agendada = pacientes que realizaram 1 ou mais consultas médicas agendadas no período, com qualquer número de consultas de $\mathrm{PA}$.

*** Pronto-atend. exclusivo = pacientes que realizaram apenas consultas médicas de PA.

Nota: A distribuição é significativamente não homogênea.

Tabela 7. Distribuição percentual de pacientes hipertensos exclusivos, segundo idade e sexo por uso do serviço. Periodo de junho de 1990 a maio de 1991.

\begin{tabular}{llccr}
\hline \multicolumn{5}{c}{ Uso do serviço } \\
\hline Idade & Sexo & $\begin{array}{c}\text { Consulta } \\
\text { agendada }\end{array}$ & $\begin{array}{c}\text { Pronto-aterd. } \\
\text { exclusivo }\end{array}$ & Total \\
\hline 12 a 20 & Masc. & 0,5 & - & 0,4 \\
& Fem. & 0,3 & - & 0,2 \\
20 a 49 & Masc. & 7,8 & 14,3 & 9,1 \\
& Fem. & 32,2 & 36,2 & 33,0 \\
50 a 59 & Masc. & 4,1 & 11,0 & 5,4 \\
& Fem. & 18,9 & 15,4 & 18,2 \\
60 e + & Masc. & 7,8 & 6,6 & 7,6 \\
& Fem. & 28,4 & 16,5 & 26,1 \\
\hline Total \% & Masc. & 20,3 & 31,9 & 22,6 \\
parcial & Fem. & 79,7 & 68,1 & 77,4 \\
\hline Total \% & & 100,0 & 100,0 & 100,0 \\
número & & 370 & 91 & 461 \\
\hline
\end{tabular}

scxo e uso do serviço é significativamente não homogênea para o conjunto dos pacientes Hipertensos Exclusivos, onde os homens usam menos freqücntemente o atendimento agendado $\mathrm{e}$ as mulhercs realizam uso mais intenso do atendimento agendado $\mathrm{cm}$ relação ao uso exclusivo do PA. Este fenômeno, porćm, está localizado apenas na faixa de 50 a 59 anos. Ainda na Tabela 7, observa-se que o sub-grupo de indivíduos atendidos apenas em PA, é mais jovem e com mais indivíduos do sexo masculino. Para esses indivíduos, em princípio "não aderentes" ao atendimento programático, seria interessante aprofundar estudo que pudesse indicar relações entre o uso do serviço (ou aderência a ações programáticas) e certas situações de vida social e de morbidade.

Cabe assinalar, ainda, que parte desta "percla" de indivíduos que não chegam a obter o atendimento programático é decorrente da falta de encaminhamento adequado para essas açōcs (o profissional médico que realizou $o$ atendimento não encaminhou o indivíduo hipertenso para o seguimento programático), c não somente de uma recusa ao atendimento. Essa "perda" por falha do profissional pôde ser detectada cm cxame de prontuários realizado após a análisc inicial dos dados aprescntados no prescnte trabalho.

\section{Discussão}

Foram abordados dois tipos de qucstōcs: primciramcnte, as relativas à captação de indivíduos portadores de alguns fatores de risco para DCV para assistência médica individual, $\mathrm{c}, \mathrm{cm}$ seguida, questōes relativas ao atendimento realizado aos individuos que freqüentaram o scrviço.

O "enfoque de risco", ao propor a deteç̧ão de indivíduos considerados de risco para DCV, deve captar para a assistência médica grupos de pessoas $\mathrm{cm}$ sua maior parte assintomálica, o que determina dificuldades não somente na identificação desses indivíduos como tambćm no postcrior controlc de saúde atravćs de assistência médica individual. A identificação de parte desses indivíluos é possível apenas cm situações de procura espontânca ao scrviço de saúde por outros motivos, quando, então, detecta-se nível pressórico elevado. Um outro grupo de indivíduos, que não frcqüenta serviço de saúde, ou o $\mathrm{faz} \mathrm{em}$ condiçōes de atendimento muito sumário, não tem a situação de risco detectada. Assim, opera-se com uma baixa cobertura na identificação de indivíduos expostos ao fator de risco para $D C V$, como também com perda de indivíduos reconliccidos como "de risco", que não adcrem ao controle de saúde. 
Quanto à qucstão dos indivíduos considerados como "de risco" para DCV, o rastreamento fazse apenas a partir dos níveis tensionais, sendo os outros fatores (tais como hipercolesterolemia, tabagismo, obesidade, diabete) considerados somente em momento posterior ao diagnóstico de hipertensão arterial. Porém, é sabido que esses outros fatores, mesmo na ausência de hipertensão arterial, contribuem para a mortalidade por DCV.

Além do problema da captação de individuos "de risco" para DCV é necessário considerar também as possibilidades efetivas de manter esse grupo de indivíduos sob permanente controle individual de saúde. Os resultados do presente trabalho sugerem haver maior dificuldade na obtenção de "aderência" ao serviço por parte dos indivíduos portadores apenas de fatores de risco, provavelmente sem doença sintomática concomitante. Sendo assim, toma-se necessário conhecer o que determina a procura e a "aderência" desses indivíduos ao serviço.

Ainda que se considere esta relativa menor "aderência" por parte desses indivíduos assintomáticos, pode-se afirmar que, no presente cstudo, o padrão de comparecimento ao serviço para consultas médicas periódicas foi, no geral, satisfatória. Isto significa que, cmbora do ponto de vista populacional possa-se esperar apenas um impacto mínimo na redução da morbimortalidade por DCV, é provável que haja benefício real para os indivíduos que efclivamente foram captados e seguem adcridos ao controle de saúde. Resta saber qual é a eficácia da aborda$\mathrm{gem}$ individual para controle de alguns fatores de risco, bem como qual o bencfício real para scu portador, em termos de qualidade de vida, notadamente na situação em que se prioriza para captação um único fator de rísco - o nível de pressão artcrial clevado.

SALA, A. et al. [Evaluation of management of paticnts with chronic degenerative diseases in a primary health clinic]. Rev. Saúde Pública, 27: 463-71, 1993. The efficacy of health actions, related to arterial hypertension and used as a strategy to decrease morbimortality due to cardiovascular diseases, in accordance with the "risk approach" and carricel out in a Primary Health Care Clinic is assessed. These actions are based on the detection of arterial hypertension in the adult population attended at the Clinic and on the control of blood pressure levels in hypertensive individuals in which other known risk factors continue to be controlled, as well as on further treatment of eventual complications. Data relating to the 3,793 patients who wcre attended at least once by doctors of the adult sector of a training health-center located in S. Paulo county (Brazil) during the period from June 1990 to
May 1991, inclusive, were evaluated. This evaluation was made according to each diagnosis undertaken as well as to the concentration of each type of consultation whether occasional, or follow-up. Of these 3,793 patients analysed, 839 presented arterial hypertension and/or diabetes, and were grouped into four categories: the exclusively hypertensive, the hipertensive with other associated chronic diseases (except diabetes), the diabetic and the diabetic with arterial hypertension. The results of this study brougth the following aspects to light: 1) The low coverage of hypertensive individuals and diabetics being attended by the health service when only the population attended by the health service is taken into consideration. 2) The incidence of patients diagnosed as hypertensive in occasional consultations who did not retum to the health service for medical follow-up indicates the difficulties involved in attracting such individuals permanently. This loss is due to both the non-appearance of patients at the consultations programmed for their follow-up as well the lack of the follow-up program on the part of the health service. 3 ) With regard to these that fulfilled the follow-up program, the concentration of medical consultations and the concentration of absences presented satisfactory proportions, compatible with the proposal of quartely medical consultations. 4) The category of exclusively hypertensive individuals presented a lower concentration of attendance at consultations and a higher proportion of absences per consultation planned than did the others categories. Finally, the limitations of the actions based on the risk approach for the control of Chronic Degenerative Diseases are discussed.

Keywords: Outcome and process assessment (health care). Hypertension, prevention. Program evaluation. Primary health care.

\section{Referências Bibliográficas}

1. CASTANHEIRA, E.R.L; TEIXEIRA, R.R.; LLMA, A.M.M. Envelhecimento e ação programática: da necessidade à construção de um objeto. In: Schraiber, L.B. Progra. mação em saúde hoje. São Paulo, Hucitec, 1990. p. 183-226. (Saúde em Debate, 30).

2. COMISS $̃$ O COORDENADORA CENTRAL DO ESTU. DO MULTICÊNTRICO DA PREVALÊNCIA DO DI. ABETES MELLITUS NO BRASIL. Estudo multicêntrico sobre a prevalência do Diabetes Mellitus no Brasil. Inf. Epidemiol. SUS, 1(3): 45-73, 1992.

3. DONABEDIAN, A. Exploration in quality assessment and monitoring. Michigan, Health Administration Press, 1980. v. 1 .

4. GONÇALVES, R.B.M. Práticas de saúde: processo de trabalho e necessidades. São Paulo, CEFOR, 1992. (Cademos CEIOR - Série textos, 1).

5. MLISTÉRIO DA SAUDE. Normas técnicas para o programa nacional de educação e controle da hipertensäo ar. terial (PNECIIA). Brasilia, Centro de Documentação, 1988.

6. MNISTÉRIO DA SAÚDE. Secretaria Nacional de Programas Especiais. Divisão Nacional de Doenças CrônicoDegenerativas. Bases estratégicas e operacionais. Brasilia, 1986. [mimeografado]. 
7. ROSE, G. Individuos enfermos y poblaciones enfermas. In: Buck, C. et al. El desafio de la epidemiologia: problemas y lecturas seleccionadas. Washington, D.C. Organización Panamericana de la Salud, 1988. p. 900-9 (OPAS - Publicación científica, 505).

8. SALA, A. A avaliação de programas de saúde. In: Schraiber, L.B. Programação em saúde hoje. São Paulo, Hucitec,
1990. p. 117-38. (Saúde em Debate, 30).

9. SCHRAIBER, L.B. Programação em saúde hoje. São Paulo, Hucitec, 1990. (Saúde em Debate, 30).

Recebido para publicaçäo em 26.11.1992

Reapresentado em 13.9.1993

Aprovado para publicação em 21.10.1993 